\title{
Predominance of ground over ceiling surfaces in binocular rivalry
}

\author{
Kerem OzKan and Myron L. Braunstein \\ University of California, Irvine, California
}

\begin{abstract}
The superiority of ground surfaces over ceiling surfaces in determining the representation of the visual world, demonstrated in several studies of visual perception and visual search, has been attributed to a preference for top-away projections resulting from ecological constraints. Recent research on binocular rivalry indicates that ecological constraints affect predominance relations. The present study considered whether there is a difference in predominance between ground and ceiling surfaces. In Experiment 1, we examined whether a ground surface would dominate a ceiling surface when one surface was presented to each eye. In Experiment 2, we used an eye-swapping paradigm to determine whether a ground surface would come to dominance faster than a ceiling surface when presented to the suppressed eye. The eye-swapping paradigm was used again in Experiment 3, but the ground and ceiling planes were replaced with frontal planes with similar variations in texture density. The results of these experiments indicate that ground surfaces are predominant over ceiling surfaces, with this predominance affecting both the dominance and suppression phases of binocular rivalry. This superiority of ground planes is independent of image properties such as the increase or decrease in texture density from the lower half to the upper half of the images.
\end{abstract}

When dissimilar images are presented to the two eyes, visual awareness may fluctuate between the two images, resulting in the perception of one image at a time rather than both images fused. This phenomenon is known as binocular rivalry (Wheatstone, 1838). The image perceived at a given moment in time is referred to as the dominant image, the other image as the suppressed image. Where in the visual hierarchy the competition between two dissimilar images is resolved is still a debated issue (Blake \& Logothetis, 2002). Fundamental to this debate is the issue of whether rivalry takes place over eye-based representations as a result of low-level interactions between monocular channels, or over stimulus-based representations as a result of competing visual representations at higher brain areas (Lee \& Blake, 1999; Logothetis, Leopold, \& Sheinberg, 1996). Recent studies suggest that binocular rivalry arises as a result of distributed processes occurring at different levels of the visual pathway (Freeman, 2005; Nguyen, Freeman, \& Alais, 2003; Ooi \& He, 2003; Wilson, 2003).

Since Levelt's (1965) observation that suppression durations are influenced by stimulus strength, various image properties that affect binocular rivalry have been identified (for a comprehensive review, see Blake, 2001). Evidence about the interaction between stimulus strength and dominance and suppression durations led to the so-called "bottom-up" theory of binocular rivalry. Blake (1989) formalized a bottom-up model in which inhibitory connections between monocular channels determined perceptual alternations in binocular rivalry. Recent fMRI studies found that interocular competition was resolved in the monocular neurons in the blind spot (Tong \& Engel, 2001) and in V1 (Polonsky, Blake, Braun, \& Heeger, 2000), or even in the lateral geniculate nucleus (LGN; Haynes, Deichmann, \& Rees, 2005; Wunderlich, Schneider, \& Kastner, 2005), supporting Blake's (1989) model.

There is contradictory evidence, however, supporting stimulus-based representations. Using single-unit recordings from monkeys, Leopold and Logothetis (1996) showed that perception-dependent activation increases at higher levels in the visual cortex with little activation in monocular neurons within V1. In addition, when Logothetis et al. (1996) flickered images on and off at $18 \mathrm{~Hz}$ while images were switching between the eyes of their observers every $333 \mathrm{msec}$, they found that their observers experienced stable percepts with temporal dynamics similar to those in conventional rivalry experiments. As a result, Logothetis et al. proposed a model in which stimulus representations would compete for dominance independently of the eye in which they are presented. Other researchers have reported that spatially nonuniform images presented to the two eyes can alternate as uniform shapes in the observer's perception. This is called interocular grouping (Kovacs, Papathomas, Yang, \& Fehér, 1996). The alternation between stimulusbased representations found with interocular grouping cannot be explained by the competition between monocular neurons proposed in eye-based explanations.

Similarly, a recent approach considers binocular rivalry to be an extension of normal binocular vision, in which 3-D surface representation mechanisms govern the dynamics of binocular rivalry by inhibiting false matches

M. L. Braunstein, myron.braunstein@uci.edu 
between the two eyes according to the same ecological constraints (Ooi \& He, 2005). As a part of this approach, the effects of surface properties such as natural boundary contours (Ooi \& He, 2006) or the coherence of surfaces (Ooi \& He, 2003) have been shown to establish dominance relations. For example, homogeneous or continuous surfaces in color dominate discontinuous images when they are presented dichoptically (Ooi \& He, 2003).

It has been shown that quantitative image properties of a stimulus, such as contrast level, have an impact on dominance durations (Blake, 2001). In addition to image properties, qualitative differences between two images that affect higher order representations have been shown to affect the probability of dominance during binocular rivalry. For example, a recent study showed that direction of motion has an impact on predominance relations, with expanding (looming) contours dominating contracting or (receding) contours (Parker \& Alais, 2007). In an earlier study, upright faces had been found to dominate inverted faces (Engel, 1956).

The present study examines the role of ground surfaces in binocular rivalry. Increasing experimental evidence shows that a background surface, especially a ground surface, provides crucial information to the visual system about the external world. The role of the ground surface in determining the perceived distance of objects was a major component of Alhazen's (ca. 1039/1989) theory of depth perception. Gibson (1950) emphasized the role of the ground surface in the perception of the visual world. He showed that optical contact with the ground surface can determine the perceived position of an object in a 3-D scene. Recent studies of the importance of ground surfaces in representing the external world have examined the role of mediated contact relations for objects not in direct contact with the ground (Meng \& Sedgwick, 2001) and the effect of surface continuity (Feria, Braunstein, \& Andersen, 2003; Sinai, Ooi, \& He, 1998). Gibson attributed the special role of ground surfaces to two main causes: (1) Humans are terrestrial creatures that rely on ground surfaces for locomotion, and (2) the ground plane is a universal property of our living habitat. As Gibson mentioned, ground surfaces are universal, whereas ceilings are mostly artifacts of human culture.

The superiority of ground planes over ceiling planes in perceptual representation has been demonstrated in several recent studies. Bian, Braunstein, and Andersen (2005) showed that the ground surface compared with a ceiling surface plays a dominant role in determining perceived distance, although Dilda, Creem-Regehr, and Thompson (2005) found accurate blind walking to targets on the ceiling. McCarley and He (2000) found a similar dominance in visual search. According to their suggestion, the visual system increases its efficiency by preferential encoding of ground surfaces. Bian and Andersen (2006) reported that ground surfaces are superior to ceiling surfaces in a changedetection task. Imura and Tomonaga (2007) reported that in both chimpanzees and humans, visual search is faster on ground-like surfaces than on ceiling surfaces, suggesting that the ground dominance effect is not a cognitive strategy unique to humans but part of evolution in visual perception.
If there is an "asymmetry of the perceptual organization" that favors ground-like surfaces (McCarley \& He, 2000), it is important to determine whether a predominance relation exists between ground surfaces and ceiling surfaces during binocular rivalry. Such a difference in predominance between ground and ceiling surfaces would be consistent with the effect of ecological constraints in binocular rivalry, as discussed by Ooi and $\mathrm{He}$ (2005).

The first experiment reported here examined predominance rates for ground and ceiling surfaces. A control experiment considered whether perceived slant might be a factor in determining these rates. The second experiment used an eye-swapping technique to compare latencies for achieving dominance in the suppressed eye for ground and ceiling surfaces. In Experiment 3, the eye-swapping technique was used with planar surfaces with similar average texture densities in the top and bottom halves of the displays to determine whether the results with ground and ceiling planes could be explained by 2 -D image variations. Overall, the results of these experiments indicate that ground surfaces are predominant over ceiling surfaces, with this predominance affecting both the dominance and suppression phases of binocular rivalry.

\section{EXPERIMENT 1 \\ Ground Versus Ceiling Predominance Rates}

In Experiment 1, we looked at binocular rivalry between ground and ceiling planes. Previous results demonstrating ground dominance in a variety of tasks, cited above, suggest that information processing is more efficient for ground planes than for ceiling planes. In Experiment 1, we considered whether ground dominance could also be observed in a rivalry paradigm.

\section{Method}

Observers. The observers were 10 undergraduate students at the University of California, Irvine. They were naive regarding the purpose of the experiment, and all had visual acuity of 20/40 or better (measured with a Snellen eye chart). All received course credit for their participation. Informed consent was obtained from all observers prior to the experiment.

Stimuli. The stimuli consisted of black-and-white checkerboard planes that resembled ground and ceiling surfaces with $86.6^{\circ}$ of slant ${ }^{1}$ (see Figure 1). The only difference between the two surfaces studied was the perspective information, with the ground surface showing convergence from bottom to top and the ceiling surface showing convergence from top to bottom. The checkerboard planes $\left(2^{\circ}\right.$ high $\times$ $4^{\circ}$ wide $)$ were centered vertically within a black rectangle $\left(8^{\circ} \mathrm{high} \times 4^{\circ}\right.$ wide) presented against a gray background. The black-and-white areas in the checkerboard pattern had luminances of 0.1 and $90.0 \mathrm{~cd} / \mathrm{m}^{2}$, respectively, resulting in a Michelson contrast of .998. The black rectangles were used to stabilize the alignment of the images of ground and ceiling planes. Red fixation circles $\left(0.5^{\circ}\right.$ in diameter) appeared within the center of the dichoptically presented ground and ceiling planes to control for eye movement artifacts during rivalry.

Apparatus and Procedure. The rivalry displays were generated and presented using MATLAB software with the Psychophysics Toolbox. Ground and ceiling planes were presented, one on each side of a gamma-corrected Sony $21-$ in. $(53-\mathrm{cm})$ flat-screen CRT monitor with $1,024 \times 768$ pixel resolution and a $90-\mathrm{Hz}$ frame refresh rate. Observers looked at the displays through a mirror stereoscope adjusted for each observer to achieve full fusion. Viewing distance was $57 \mathrm{~cm}$. A chinrest was mounted to maintain head stability. 


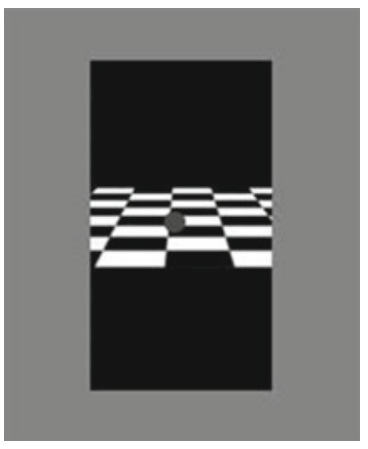

Left Eye

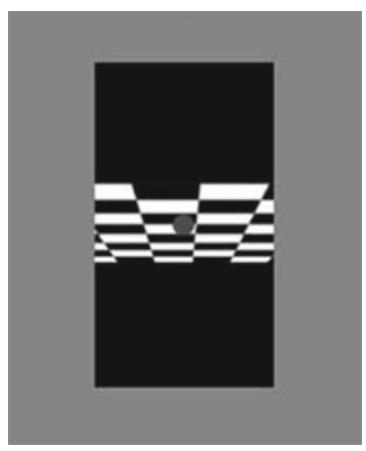

Right Eye
Figure 1. Stimuli used in Experiments 1 and 2.

The observers viewed the stimulus in twelve 60 -sec trials $(6$ repeats $\times 2$ left vs. right eye) during one session, preceded by a 90 -sec practice block. Between the trials, a gray screen appeared for $10 \mathrm{sec}$, indicating the end of the trial. During the trials, the observers' task was to report their percept continuously using one of the three buttons, indicating complete dominance of ground, complete dominance of ceiling, or a blend thereof or piecemeal rivalry. The third button was included because blend or piecemeal percepts are frequently observed during rivalry. The observers were asked to maintain fixation while they were performing the task. The duration of buttonpresses was recorded separately for each percept. The experiment was run in a dark room.

\section{Results}

The dependent variable in Experiment 1 was the predominance rate for ground and ceiling planes. Predominance rates were calculated for each observer in each trial by dividing the dominance duration for each stimulus by the total time for that trial. These rates were then averaged across trials for each observer. Predominance rates averaged across observers are shown in Figure 2. The proportion of piecemeal percepts was .10. Proportions of piecemeal percepts have been reported in the range of .1 to .2 for a variety of rivalry stimuli (e.g., de Weert, Snoeren, \& Koning, 2005; Ooi \& He, 2006; Parker \& Alais, 2007).

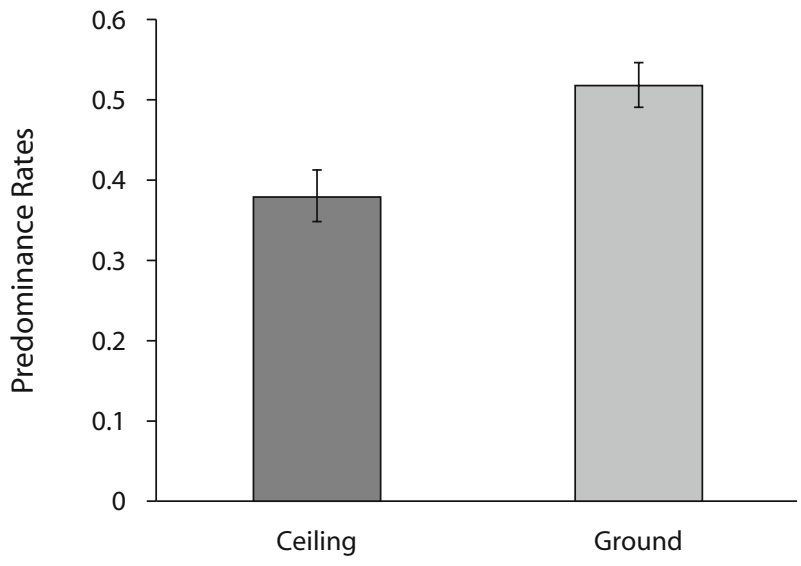

Figure 2. Predominance rates of ground and ceiling surfaces in Experiment 1. Error bars represent \pm 1 standard error.
There was significantly higher predominance for ground surfaces than ceiling surfaces [paired-sample $t$ test; $t(9)=$ $2.72, p<.05]$. This result supported our hypothesis that ground surfaces tend to dominate ceiling surfaces in binocular rivalry.

\section{CONTROL EXPERIMENT}

The results of Experiment 1 suggest a bias to perceive ground surfaces during binocular rivalry. A possible alternative explanation of the results, however, is that there is a bias to perceive surfaces that appear more frontoparallel and that the ground surfaces appeared more frontal. For this reason we conducted a control experiment, using a paired comparison method, to determine whether observers judged ground surfaces to be more frontal than ceiling surfaces.

\section{Method}

Observers. The observers were 8 undergraduate students at the University of California, Irvine. They were naive regarding the purpose of the experiment and none had participated in any other experiment in this study. All had visual acuity of 20/40 or better (measured with a Snellen eye chart), and all received course credit for their participation. Informed consent was obtained from all observers prior to the experiment.

Stimuli. The stimuli consisted of black-and-white checkerboard planes representing ground and ceiling surfaces, each with three different slants: $85.0^{\circ}, 86.6^{\circ}$, and $88.3^{\circ}$ (see Figure 3 ). The middle slant level was the same as the slant used in Experiment 1. The checkerboard planes $\left(2^{\circ} \times 4^{\circ}\right)$ were centered vertically within a black rectangle $\left(8^{\circ} \times 4^{\circ}\right)$ presented against a gray background. Red fixation circles $\left(0.5^{\circ}\right.$ in diameter $)$ appeared within the center of the ground and ceiling planes. Unlike in the rivalry experiments, ground and ceiling planes were presented separately, not dichoptically.

Procedure. The apparatus and viewing conditions were the same as in Experiment 1, except that observers viewed the displays monocularly through the mirror stereoscope. Although the images were presented separately, observers viewed the displays through the mirror stereoscope in order to create the same viewing conditions as in the rivalry experiments. The observers were asked to each use whichever eye was better to look at the images and were instructed to use the same eye throughout the experiment. An eye patch was worn over the other eye.

Each trial started with the presentation of a fixation point for $5 \mathrm{sec}$. This was followed by a fixation point and either a ground or a ceiling image for $10 \mathrm{sec}$; then either a ceiling or ground scene with the same slant as in the first frame was presented for $10 \mathrm{sec}$. The observers' task was to indicate, using the mouse buttons, whether the first or the second image was slanted more. There were 60 experimental trials consisting of 10 repetitions of six conditions ( 3 slant levels $\times 2$ orders: ground first or ceiling first), preceded by 15 randomly selected practice trials.

\section{Results}

Figure 4 shows the proportion of trials on which each surface was selected as more frontal, for the three slants. As seen in the graph, in all three slants our observers selected ceiling planes as being more frontal than ground planes. The proportion of choosing the ceiling planes across the six conditions was $.63(S D=.05)$. Paired-sample $t$ tests conducted to compare the proportions of choosing a ground surface and a ceiling surface for each slant level found no significant differences $(p>.05)$. 


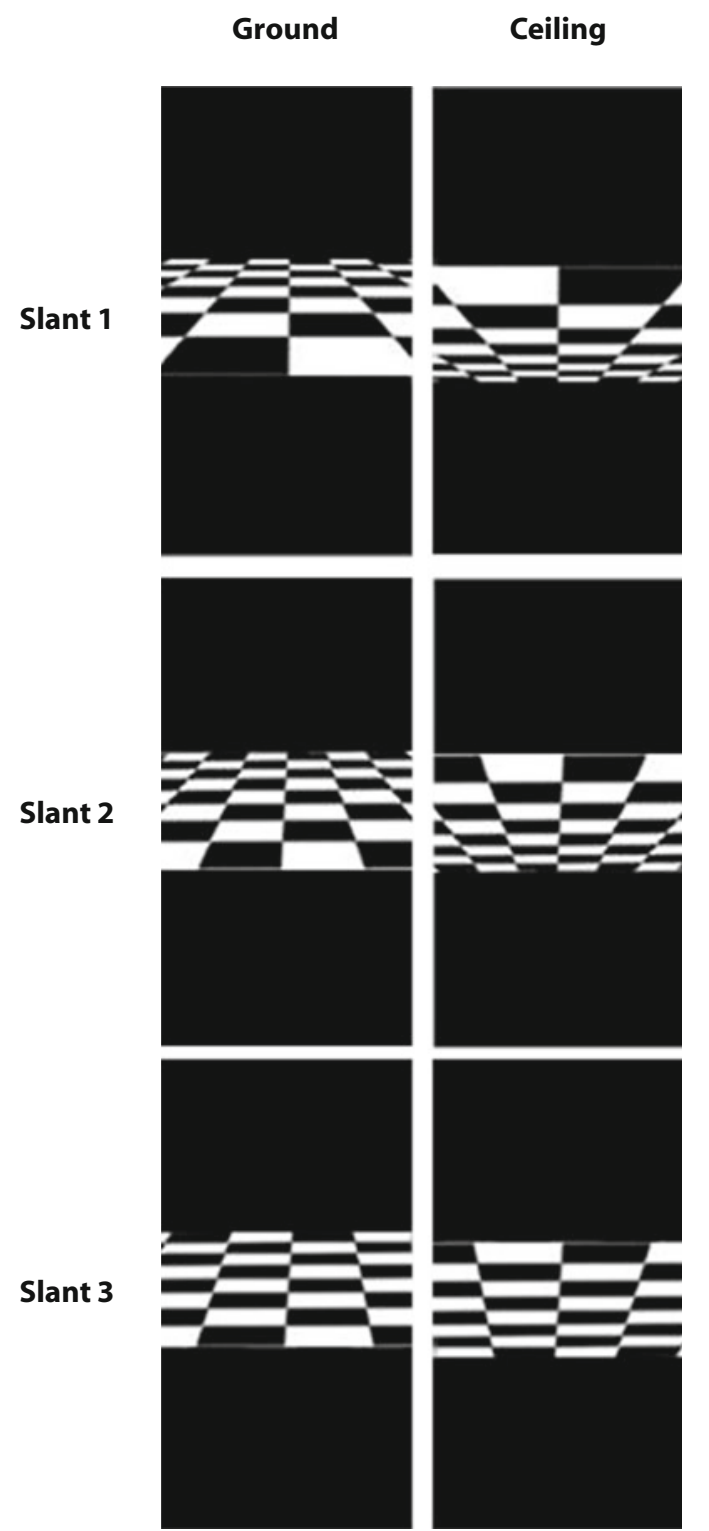

Figure 3. Stimuli used in the control experiment: black-andwhite checkerboard planes representing ground and ceiling surfaces with three different slants.

These results do not support the possibility that ground planes are perceived as being more frontal than ceiling planes, indicating that the higher predominance rates for ground planes found in Experiment 1 cannot be explained by a tendency to perceive ground planes as being more frontal. Although the proportions failed to reach significance, the trend for all three slant levels was to choose the ceiling planes as being more frontal than the ground planes.

\section{EXPERIMENT 2 Eye Swapping}

Previous research showed that it is the eye of origin, rather than specific stimulus properties, that is suppressed

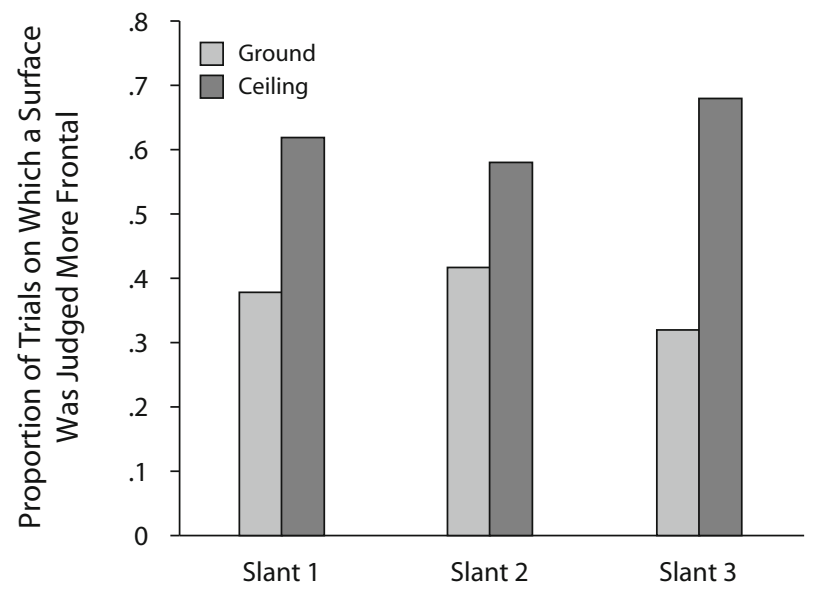

Figure 4. The proportion of trials on which the ceiling or ground surface was selected as more frontal for each of the three slants in the control experiment.

during binocular rivalry (Blake, Westendorf, \& Overton, 1980). Even drastic changes in the stimuli are unseen while the image is presented to the suppressed eye (Blake, Yu, Lokey, \& Norman, 1998). These psychophysical experiments, together with recent imaging studies (e.g., Tong \& Engel, 2001; Wunderlich et al., 2005), have supported the eye-of-origin hypothesis of binocular rivalry.

Recent studies, however, show that under certain conditions high-level information is still processed during the suppression phases of binocular rivalry. Jiang, Costello, and He (2007) showed that highly familiar images tend to gain dominance faster when presented to the suppressed eye. This suggests that during the suppression phases of binocular rivalry, high-level form information from the unseen image reaches a level of representation which affects the salience of the stimulus. Several neuroimaging studies also support the idea that high-level information can be processed during suppression. Williams, Morris, McGlone, Abbott, and Mattingley (2004) showed that emotional faces generate higher amygdala activation than neutral faces during the suppression phase of binocular rivalry. If the perceptual salience of the images affects recovery from suppression, we would expect more rapid recovery with ground than with ceiling surfaces. Therefore, in Experiment 2, we tested whether a ground surface would come to exclusive dominance faster than would a ceiling surface, when presented to the suppressed eye.

\section{Method}

Observers. Observers were 5 undergraduate students at the University of California, Irvine. They were naive regarding the purpose of the experiment and none had participated in any other experiment in this study. All had visual acuity of 20/40 or better (measured with a Snellen eye chart) and all received course credit for their participation. Informed consent was obtained from all observers prior to the experiment.

Stimuli. The stimuli consisted of black-and-white checkerboard planes that resembled ground and ceiling surfaces (see Figure 1). The checkerboard planes $\left(2^{\circ} \times 4^{\circ}\right)$ were centered vertically within 


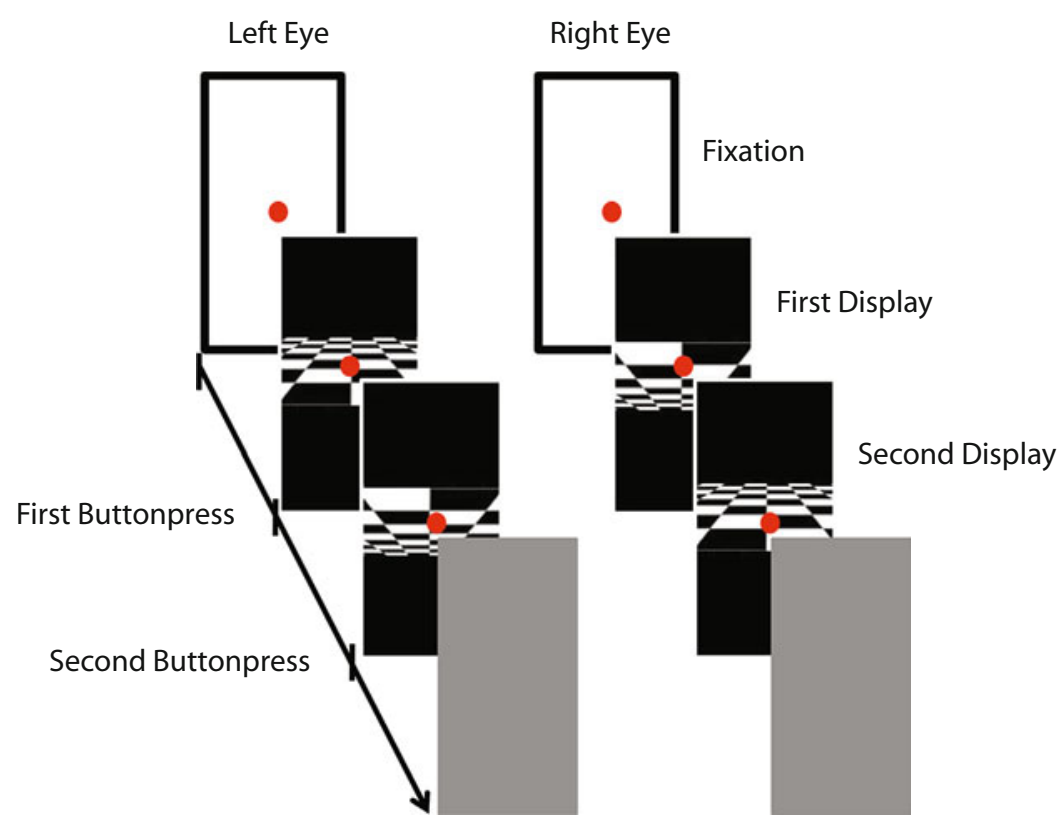

Figure 5. Design of trials in Experiment 2. Which display was presented first to each eye was counterbalanced.

a black rectangle $\left(8^{\circ} \times 4^{\circ}\right)$ presented against a gray background, as in Experiment 1. Red fixation circles $\left(0.5^{\circ}\right.$ in diameter $)$ appeared within the center of the dichoptically presented ground and ceiling planes to control for eye-movement artifacts. The apparatus was the same as in Experiment 1.

Procedure. This experiment used an eye-swapping procedure developed by Blake et al. (1980) and used by Lee and Blake (2004). The design of the trials is shown in Figure 5. Observers viewed the same rivalrous figures as in Experiment 1. They were told to press a button when they saw one of the figures exclusively (either the ground surface or the ceiling surface, in separate blocks of trials). Following the buttonpress, the images were swapped between the eyes, so that the dominant figure was presented to the suppressed eye and the suppressed figure was presented to the dominant eye. After this exchange of images, the observers' task was to press the button again to indicate exclusive dominance of the same figure tracked before the swap. Following the second buttonpress, the gray screen appeared for $10 \mathrm{sec}$, indicating the end of the trial. Following Lee and Blake's (2004) procedure, we swapped the images while decreasing the contrast to zero and then gradually increased it back to the original level, with the contrast change following a sinusoidal distribution to eliminate rapid transients. The total duration of contrast change following the swap of the images was $150 \mathrm{msec}$.

Observers participated in two blocks of trials, one in which they were to report dominance of the ground surface, and one in which they were to report dominance of the ceiling surface. Each block had 50 ( 25 repeats $\times 2$ left vs. right eye) trials. The order of the blocks was counterbalanced across observers. Eye order was randomized between trials. Latencies for the reappearance of the target image after the swap were recorded. Since eye order was not a significant factor, scores were averaged between eyes for each observer.

\section{Results}

Figure 6 shows reappearance latencies for reporting ground and ceiling surfaces, when presented to the suppressed eye, for each observer. Our results show that the ground surface became dominant more quickly than the ceiling surface when both were presented to the suppressed eye; this was true for all 5 observers. A paired-sample $t$ test across observers found reappearance latency for ground surfaces to be significantly lower than for ceiling surfaces $[t(4)=4.28, p<.05]$. In contrast to this result, latencies for exclusive perception of ground and ceiling surfaces in the initial exposure were not significantly different from each other. Our results show that ground surfaces emerge to dominance faster than ceiling-like surfaces when presented to the suppressed eye after the swap of images.

As noted in previous research (Blake et al., 1980), when the dominant image was presented to the suppressed eye, it disappeared from conscious perception for a while. Latency

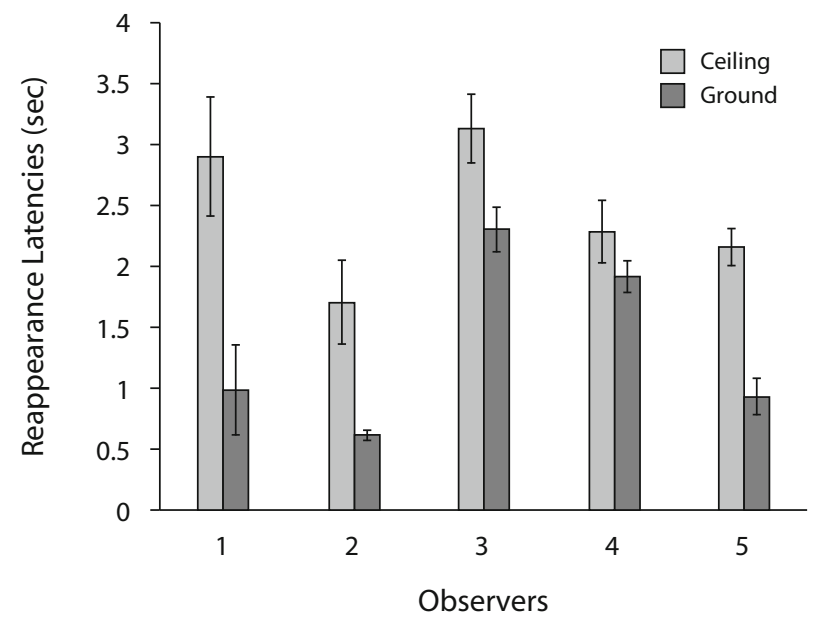

Figure 6. Reappearance latencies of ground and ceiling surfaces after the swap of images for each observer in Experiment 2. Ground surfaces became dominant faster than ceiling surfaces in the suppressed eye. Error bars represent \pm 1 standard error. 
for reappearance averaged across observers was $1.9 \mathrm{sec}$ $(S D=0.84 \mathrm{sec})$. Since there was a considerable amount of time between the swap and the return to dominance of the target figure, the inhibition system in the monocular channels appears to have a clear impact on rivalry. Because the observer's task was to press a button again when the target stimulus was exclusively dominant, average latency for reappearance includes the duration for perceiving any piecemeal rivalry that occurred after the swap of images.

\section{EXPERIMENT 3 \\ Eye Swapping With 2-D Images}

In Experiment 3, we considered whether the effect found in Experiment 2 could be due to 2-D image properties rather than 3-D differences between ground and ceiling planes. Ground surfaces show increasing compression from bottom to top, whereas ceiling surfaces show increasing compression from top to bottom. As a result, in the ground planes spatial frequency increases from bottom to top, whereas in the ceiling planes spatial frequency increases from top to bottom. In Experiment 3, we considered whether the shorter reemergence time for ground surfaces in Experiment 2 could be due to differences in the spatial frequency distribution between the two types of surface rather than to differences in 3-D perspective information.

In order to test this, we created frontoparallel displays with only two spatial frequencies in each: either a higher spatial frequency pattern at the top, as in a ground plane, or a higher spatial frequency pattern at the bottom, as in a ceiling plane. The use of only two spatial frequencies was intended to remove the texture gradient information for perceived slant that was present in the ground and ceiling planes in the previous experiments, so that the new planes would be perceived as 2-D. As a result, in Experiment 3, we tested whether the effect found in Experiment 2 was due to perceptual differences between ground and ceiling planes or due to differences in image statistics that might affect low-level processing.

\section{Method}

Observers. Observers were 5 undergraduate students at the University of California, Irvine. All observers met the same visualacuity requirement as in the previous experiments. All were naive regarding the purpose of the experiment and none had participated in any other experiments in this study. The observers received course credit for their participation.

Stimuli. The stimuli consisted of black-and-white checkerboard patterns (see Figure 7). The top and bottom halves of each display had different spatial frequencies, but the spatial frequency did not vary within those regions. The top half of a display had a spatial frequency equal to that found at a point either one third of the way from the top of a ceiling display (Display 1) or one third of the way from the top of a ground display (Display 2) in the previous experiments. The bottom half of each display had spatial frequencies taken at a point one third of the way from the bottom of a ceiling display (Display 1) or one third of the way from the bottom of a ground display (Display 2). Replacing the texture compression gradients with a step function was intended to remove the perception of the displays as ground or ceiling planes. The checkerboard planes $\left(2^{\circ} \times 4^{\circ}\right)$ were centered vertically within a black rectangle $\left(8^{\circ} \times 4^{\circ}\right)$ presented against a gray background, as in the first two experiments.

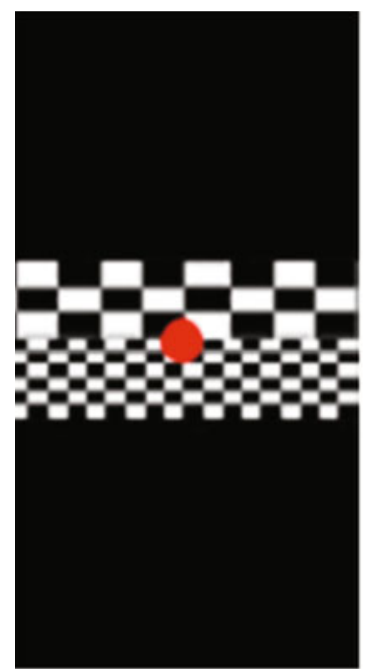

Display 1

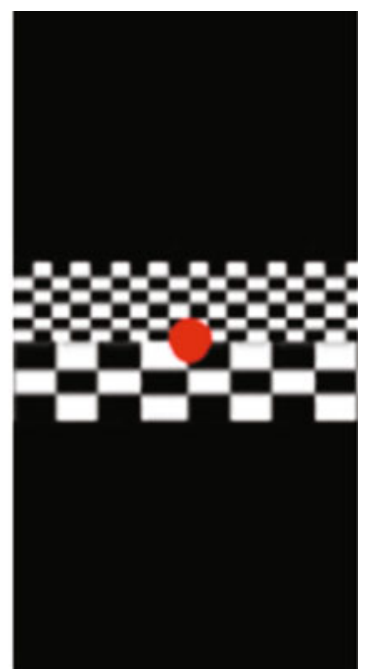

Display 2
Figure 7. Stimuli used in Experiment 3.

The spatial frequencies of the two checkboard patterns were $1 \mathrm{cpd}$ and $2.25 \mathrm{cpd}$, respectively. Red fixation circles $\left(0.5^{\circ}\right.$ in diameter $)$ appeared within the center of the dichoptically presented ground and ceiling planes to control for eye-movement artifacts.

Procedure. We used the same eye-swapping procedure as in Experiment 2 . The observers' task was to press a button when they saw one of the figures (in Figure 7) exclusively. Following the press, the images were swapped from one eye to the other. After the exchange of the images, the observers' task was to press the button again to indicate the exclusive dominance of the same figure tracked before the swap. Reappearance latencies for the two images after the swap were recorded in two separate blocks, each with 50 ( 25 repeats $X$ 2 left vs. right eye) trials. Eye order was not a significant factor, so scores were averaged between eyes for each observer.

\section{Results}

Figure 8 shows reappearance latencies for reporting planes with texture density greater at the bottom or greater at the top, when presented to the suppressed eye, for each observer. In contrast to Experiment 2, 4 of our 5 observers did not show a significant difference between the reappearance latencies for the 2-D versions of the ground and ceiling planes. This suggests that the effect found in Experiment 2 is due to 3-D differences between ground and ceiling surfaces and that the difference in spatial frequency differences between the top and bottom parts of the surfaces was not the main determinant.

As in previous research (Blake et al., 1980), as well as in Experiment 2, when the dominant image was presented to the suppressed eye it disappeared from conscious perception for a while. The mean reappearance latency was $3.61 \mathrm{sec}(S D=2.29)$. As in the previous experiment, mean reappearance latencies also included the duration for reporting any piecemeal or blend rivalry. The overall mean reappearance latency was longer than in Experiment 2. This suggests that the ground and ceiling planes in Experiment 2 overcame suppression faster than did the frontal planes in the present experiment. 


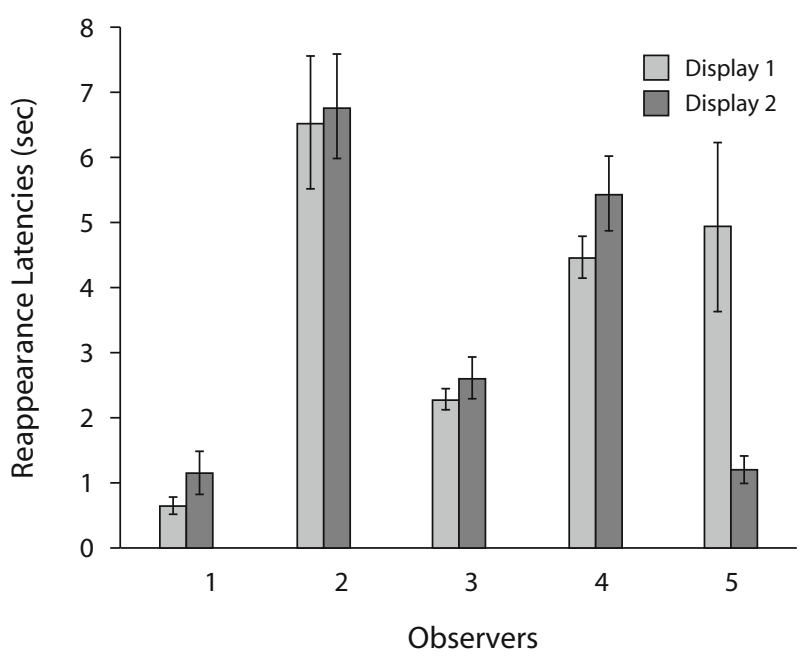

Figure 8. Reappearance latencies of two planes after the swap of images for each observer in Experiment 3. Error bars represent \pm 1 standard error.

\section{DISCUSSION}

In three experiments, we examined the differences between ground surfaces and ceiling surfaces in temporal dynamics associated with binocular rivalry. In Experiment 1 , we found a higher predominance for perceiving ground surfaces than ceiling surfaces during rivalry. In Experiment 2, using an eye-swapping technique, we found that ground surfaces took less time than ceiling surfaces to return to dominance when they were presented to the suppressed eye. In ground planes, spatial frequency increases from bottom to top, whereas in the ceiling planes, spatial frequency increases from top to bottom. In Experiment 3, we considered whether the results of Experiment 2 could be due to differences between ground and ceiling planes in 2-D image properties rather than to 3-D differences in perceptual organization. We used the same eye-swapping technique as in Experiment 2, with frontoparallel versions of the ground and ceiling surfaces. These new planes had overall 2-D image properties similar to those of the ground and ceiling planes, but had $0^{\circ}$ slant. The effects found in Experiment 2 were not found with the frontoparallel planes, suggesting that 3-D differences between ground and ceiling planes, rather than the 2-D image properties, determine the faster reappearance latencies of ground planes.

Dominance of ground surfaces in visual search (McCarley $\& \mathrm{He}, 2000)$ and in determining the perceived layout of objects (Bian et al., 2005) was discussed previously. The main difference between ground surfaces and ceiling surfaces is in the perspective information: Ground surfaces show increasing compression from bottom to top, whereas ceiling surfaces show increasing compression from top to bottom. Since humans are terrestrial creatures that move about on ground surfaces, our visual systems are adapted to operate on ground surfaces (Gibson, 1950). Our results agree with the previous studies, which proposed that information processing is more efficient on ground surfaces, suggesting that the ground dominance effect is a part of our perceptual organization (Bian et al., 2005; McCarley \& He, 2000).

Other image properties related to subjective surface formation and boundary contours have been found to affect predominance relations during binocular rivalry (Ooi \& He, 2003, 2006). Recently, de Weert et al. (2005) showed that strong gestalt figures tend to have longer dominance durations. These studies suggest that local stimulus properties and lower cortical levels are not the sole determinants of phase durations in binocular rivalry. Instead, rivalry is a complex process that involves multiple cortical areas with feedback and feedforward interactions (Alais \& Blake, 1998; Ooi \& He, 1999). It is likely that the grounddominance effect is based on perceptual attributes that affect eye dominance duration as a top-down influence.

Moreover, Bian and Andersen (2006) recently reported that ground surfaces are superior to ceiling surfaces in change detection. That study suggests a mediating role of attention in the ground-dominance effect. Stimulus-driven attention to ground surfaces can be one of the explanations of extended dominance durations in Experiment 1. Hybrid models of binocular rivalry promote the role of feedback projection to pattern-selective neurons as an account for the sustained effects of top-down influences on selective attention (Tong, Meng, \& Blake, 2006). In fact, ground dominance may represent the selective preference of our perceptual system to attend to a top-away projection rather than to a bottom-away projection as a result of some ecological constraints that developed through our evolution as terrestrial creatures. In that respect, hybrid models may explain the possible neural mechanisms underlying our findings.

Overall, in this study we demonstrated that ground surfaces dominate ceiling surfaces during binocular rivalry. Also, ground surfaces regain dominance faster than do ceiling surfaces when they are presented to the suppressed eye. Our results further support the idea that binocular rivalry is a phenomenon beyond the inhibition process in monocular neurons and that principles of perceptual organization are effective in binocular rivalry.

\section{AUTHOR NOTE}

This research was supported by NIH Grant EY18334. We thank Wilson Tao-Yen Wang for assistance with data collection and participation in discussions of this research. Correspondence concerning this article should be addressed to M. L. Braunstein, 3151 Social Science Plaza, University of California, Irvine, CA 92697-5100 (e-mail: myron .braunstein@uci.edu).

Note-Accepted by the previous editorial team, when Thomas H. Carr was Editor.

\section{REFERENCES}

Alais, D., \& BlaKe, R. (1998). Interactions between global motion and local binocular rivalry. Vision Research, 38, 637-644.

AlHaZen, I. (1989). Book of optics. In A. I. Sabra (Trans.), The optics of Ibn-Haytham (Vol. 1). London: Warburg Institute. (Original work published ca. 1039)

Bian, Z., \& Andersen, G. J. (2006). Change detection and primacy of the ground surface in scene organization [Abstract]. Journal of Vision, 6, 732a. doi:10.1167/6.6.732 
Bian, Z., Braunstein, M. L., \& Andersen, G. J. (2005). The ground dominance effect in the perception of 3-D layout. Perception \& Psychophysics, 67, 802-815.

Blake, R. (1989). A neural theory of binocular rivalry. Psychological Review, 96, 145-167.

BLAKE, R. (2001). A primer on binocular rivalry, including current controversies. Brain \& Mind, 2, 5-38.

Blake, R., \& Logothetis, N. K. (2002). Visual competition. Nature Reviews Neuroscience, 3, 1-11.

Blake, R., Westendorf, D. H., \& Overton, R. C. (1980). What is suppressed during binocular rivalry? Perception, 9, 223-231.

Blake, R., Yu, K., Lokey, M., \& Norman, H. (1998). Binocular rivalry and visual motion. Journal of Cognitive Neuroscience, 10, 46-60.

de Weert, C. M. M., Snoeren, P. R., \& Koning, A. (2005). Interactions between binocular rivalry and Gestalt formation. Vision Research, 45, 2571-2579

Dilda, V., Creem-Regehr, S. H., \& Thompson, W. B. (2005). Perceiving distances to targets on the floor and ceiling: A comparison of walking and matching measures [Abstract]. Journal of Vision, 5, 196a. doi: $10.1167 / 5.8 .196$

ENGEL, E. (1956). The role of content in binocular resolution. American Journal of Psychology, 69, 87-91.

Feria, C. S., Braunstein, M. L., \& Andersen, G. J. (2003). Judging distance across texture discontinuities. Perception, 32, 1423-1440.

Freeman, A. W. (2005). A multistage model for binocular rivalry. Journal of Neurophysiology, 94, 4412-4420.

Gibson, J. J. (1950). The perception of the visual world. Oxford: Houghton Mifflin.

Haynes, J. D., Deichmann, R., Rees, G. (2005). Eye-specific effects of binocular rivalry in the human lateral geniculate nucleus. Nature, 438, 496-499.

Imura, T., \& TomonagA, M. (2007). Visual search on the ground-like surface defined by texture gradients in chimpanzees (Pan troglodytes) and humans (Homo sapiens) [Abstract]. Journal of Vision, 7, 284a. doi:10.1167/7.9.284

Jiang, Y., Costello, P., \& He, S. (2007). Processing of invisible stimuli: Faster for upright faces and recognizable words to overcome interocular suppression. Psychological Science, 18, 349-355.

Kovacs, I., Papathomas, T. V., Yang, M., \& Fehér, A. (1996). When the brain changes its mind: Interocular grouping during binocular rivalry. Proceedings of the National Academy of Sciences, 93, 15508-15511.

LEE, S., \& BLAKE, R. (1999). Rival ideas about binocular rivalry. Vision Research, 39, 1447-1454.

LeE, S., \& Blake, R. (2004). A fresh look at interocular grouping during binocular rivalry. Vision Research, 44, 983-991.

LEOPOLD, D. A., \& Logothetis, N. K. (1996). Activity changes in early visual cortex reflect monkeys' percepts during binocular rivalry. $\mathrm{Na}$ ture, 379, 549-553.

LeVELt, W. J. M. (1965). On binocular rivalry. Oxford: Institute for Perception RVO-TNO.

Logothetis, N. K., Leopold, D. A., \& Sheinberg, D. L. (1996). What is rivalling during binocular rivalry? Nature, 380, 621-624.

McCarley, J. S., \& He, Z. J. (2000). Asymmetry in 3-D perceptual organization: Ground-like surface superior to ceiling-like surface. Perception \& Psychophysics, 62, 540-549.

Meng, J. C., \& Sedgwick, H. A. (2001). Distance perception mediated through nested contact relations among surfaces. Perception \& Psychophysics, 63, 1-15.

Nguyen, V. A., Freeman, A. W., \& Alais, D. (2003). Increasing depth of binocular rivalry suppression along two visual pathways. Vision Research, 43, 2003-2008.

OoI, T. L., \& He, Z. J. (1999). Binocular rivalry and visual awareness: The role of attention. Perception, 28, 551-574.

OoI, T. L., \& He, Z. J. (2003). A distributed intercortical processing of binocular rivalry: Psychophysical evidence. Perception, 32, 155-166.

OoI, T. L., \& He, Z. J. (2005). Surface representation and attention modulation mechanisms in binocular rivalry. In D. Alais \& R. Blake (Eds.), Binocular rivalry (pp. 117-135). Cambridge, MA: MIT Press.

OoI, T. L., \& HE, Z. J. (2006). Binocular rivalry and surface-boundary processing. Perception, 35, 581-603.

Parker, A., \& Alais, D. (2007). A bias for looming stimuli to predominate in binocular rivalry. Vision Research, 47, 2661-2674.

Polonsky, A., Blake, R., Braun, J., \& Heeger, D. J. (2000). Neuronal activity in human primary visual cortex correlates with perception during binocular rivalry. Nature Neuroscience, 3, 1153-1159.

SinaI, M. J., OoI, T. L., \& He, Z. J. (1998). Terrain influences the accurate judgment of distance. Nature, 395, 497-500.

Stevens, K. A. (1983). Slant-tilt: The visual encoding of surface orientation. Biological Cybernetics, 46, 183-195.

Tong, F., \& Engel, S. A. (2001). Interocular rivalry revealed in the human cortical blind-spot representation. Nature, 411, 195-199.

Tong, F., Meng, M., \& Blake, R. (2006). Neural bases of binocular rivalry. Trends in Cognitive Sciences, 10, 502-511.

Wheatstone, C. (1838). On some remarkable, and hitherto unobserved, phenomena of binocular vision. Philosophical Transactions of the Royal Society of London, 128, 371-394.

Williams, M. A., Morris, A. P., McGlone, F., Аввott, D. F., \& MatTINGLEY, J. B. (2004). Amygdala responses to fearful and happy facial expressions under conditions of binocular suppression. Journal of Neuroscience, 24, 2898-2904.

Wilson, H. R. (2003). Computational evidence for a rivalry hierarchy in vision. Proceedings of the National Academy of Sciences, 100, 1449914503.

Wunderlich, K., Schneider, K. A., \& Kastner, S. (2005). Neural correlates of binocular rivalry in the human lateral geniculate nucleus. Nature Neuroscience, 8, 1595-1602.

\section{NOTE}

1. Slant is defined as the angle between the line of sight and the surface normal (Stevens, 1983). A frontal plane would have a slant of $0^{\circ}$. A ground or ceiling plane, with a horizontal line of sight, would have a slant magnitude of $90^{\circ}$.

(Manuscript received December 10, 2007; revision accepted for publication March 23, 2009.) 